\title{
Prácticas de aula de los profesionales de pedagogía terapéutica para atender a la diversidad: hacia un enfoque inclusivo
}

\author{
Francisco J. García-Prieto \\ Manuel Delgado-García \\ Inmaculada Gómez-Hurtado \\ Asunción Moya Maya \\ Universidad de Huelva. España. \\ fjavier.garcia@dedu.uhu.es \\ manuel.delgado@dedu.uhu.es \\ inmaculada.gomez@dedu.uhu.es \\ asuncion@dedu.uhu.es
}

Recibido: 29/9/2020

Aceptado: 5/2/2021

Publicado: 5/7/2021

\section{Resumen}

En el presente estudio se analizan las prácticas de aula del especialista de atención a la diversidad en los centros de educación infantil y primaria (CEIP) de Andalucía. Mediante un diseño de naturaleza cualitativa de carácter transversal, se obtienen los datos con dos fuentes principales de información (entrevista semiestructurada diseñada ad hoc y observación directa y no participante) sobre una muestra de 142 especialistas. Los resultados revelan el desarrollo de prácticas inclusivas respecto al uso de estrategias didácticas y metodologías activas; la importancia de recursos plurales y funcionales; la puesta en práctica de un apoyo pedagógico coordinado o colaborativo, y la flexibilización en la dinámica y la organización de aula. También se identifican posibilidades, dificultades y limitaciones. Las conclusiones apuntan que las prácticas que están desarrollando los especialistas pretenden contribuir a la mejora de las escuelas en relación con la atención a la diversidad, siendo el apoyo colaborativo a la comunidad educativa la función principal para la promoción de una escuela inclusiva que atienda a todo el alumnado.

Palabras clave: atención a la diversidad; inclusión escolar; práctica pedagógica; apoyo y metodologías activas

Resum. Pràctiques d'aula dels professionals de Pedagogia Terapèutica per atendre la diversitat: cap a un enfocament inclusiu

En el present estudi s'hi analitzen les pràctiques d'aula de l'especialista d'atenció a la diversitat als centres d'educació infantil i primària (CEIP) d'Andalusia. Mitjançant un disseny de naturalesa qualitativa de caràcter transversal, s'obtenen les dades a partir de dues fonts principals d'informació (entrevista semiestructurada dissenyada ad hoc i observació directa i no participant) sobre una mostra de 142 especialistes. Els resultats revelen el desenvolupament de pràctiques inclusives respecte a l'ús d'estratègies didàctiques i metodologies actives; la importància de recursos plurals i funcionals; la posada en pràctica d'un suport pedagògic coordinat o col-laboratiu, i la flexibilització en la dinàmica i 
l'organització d'aula. També s'hi identifiquen possibilitats, dificultats i limitacions. Les conclusions apunten al fet que les pràctiques que estan desenvolupant els especialistes pretenen contribuir a millorar les escoles en relació amb l'atenció a la diversitat, de manera que el suport col-laboratiu a la comunitat educativa esdevé la funció principal per promoure una escola inclusiva que atengui tot l'alumnat.

Paraules clau: atenció a la diversitat; inclusió escolar; pràctica pedagògica; suport educatiu i metodologies didàctiques actives

Abstract. Classroom practices of therapeutic pedagogy professionals to attend to diversity: Toward an inclusive approach

This research analyzes the classroom practices of diversity care specialists in early childhood and primary education schools of Andalusia. A cross-sectional qualitative design was used to obtain data from two main sources: a semi-structured interview designed ad hoc and direct and non-participant observation with a sample of 142 participants. The results reveal the development of inclusive practices regarding the use of didactic strategies and active methodologies, the importance of plural and functional resources, the implementation of coordinated or collaborative pedagogical support, and flexibility in the dynamics and organization of the classroom. Opportunities, difficulties, and limitations are also identified. The conclusions indicate that the practices being developed by these specialists aim to contribute to improving schools in relation to attention to diversity, with collaborative support for the educational community being the main function for the promotion of an inclusive school that attends to all students.

Keywords: attention to diversity; inclusive education; pedagogical practice; educational support; active teaching methodologies

\author{
Sumario \\ 1. Introducción 4. Discusión y conclusiones \\ 2. Método Referencias bibliográficas \\ 3. Resultados
}

\title{
1. Introducción
}

Las investigaciones realizadas sobre atención a la diversidad sugieren un modelo educativo fijado en los principios de la inclusión escolar, teniendo como objetivo la eliminación de la exclusión social y considerando que la educación es un derecho básico y la base fundamental de una sociedad más justa y equitativa (Ainscow, 2020). La educación inclusiva conlleva un proceso de identificación y eliminación de barreras para la presencia, participación y consecución de logros de todos los estudiantes, poniendo especial atención en aquellos estudiantes que pueden estar en riesgo de marginación, exclusión o bajo rendimiento (Muntaner, Roselló y De la Iglesia, 2016). Sus principios son la igualdad, la accesibilidad, la individualidad y la democracia, persiguiendo el derecho a la educación de todos los niños y las niñas. 
En España, los cambios políticos y sociales han ido elaborando una respuesta educativa al alumnado con necesidades educativas especiales desde una perspectiva más inclusiva, relacionando la inclusión con este alumnado (Echeita, 2017), no dejando de tener en cuenta una perspectiva centrada en el modelo del déficit, existiendo un profesional concreto para la atención a la diversidad y grupos homogéneos para alumnado con necesidades específicas de apoyo educativo (Liasidou y Antoniou, 2013) y destacando una falta de formación considerable en el ámbito de la atención a la diversidad por parte de los tutores (Brownell et al., 2005). De acuerdo con Noguera y Hernández (2019), es importante la labor del maestro especialista en atención a la diversidad como colaborador de los tutores y el profesorado en los diferentes escenarios de apoyo. Dicho profesional necesita una actitud proinclusión para el desarrollo de una respuesta educativa adecuada para todo el alumnado (Collado-Sanchis et al., 2020).

Las funciones de apoyo de estos especialistas en Andalucía se desarrollan en cuatro modalidades de escolarización (aula ordinaria, aula de integración, aula específica y centro específico) (Instrucciones del 8 de marzo de 2017), en las cuales se despliegan cuatro modelos de apoyo en las que el especialista tiene un rol diferente (Biedma y Moya, 2015; Salas, 2016). Parrilla (1996) enuncia cuatro modelos básicos que actualmente siguen desarrollándose en la práctica: el modelo de apoyo terapéutico, el modelo de apoyo colaborativo/individual, el modelo de apoyo de consulta/recursos y el modelo de apoyo curricular.

En este sentido, es importante desarrollar un concepto de apoyo inclusivo en el cual el profesional de atención a la diversidad sea un agente más en pro de la inclusión escolar. La perspectiva de un apoyo inclusivo no está centrada en el alumnado con déficit, sino que más bien genera una perspectiva más amplia del mismo, sosteniendo que puede ser llevado a cabo por cualquier profesional y hacia todo el alumnado (Corujo, Gallego y Jiménez, 2018), sustentándose en los principios básicos de las escuelas inclusivas.

Este nuevo planteamiento del profesional de atención a la diversidad está ligado a una nueva forma de entender el apoyo, construyendo un modelo más inclusivo y adoptando un nuevo enfoque llamado apoyo colaborativo o comunitario (Echeita et al., 2019). A su vez, este nuevo apoyo lleva implícito el uso de estrategias didácticas activas, recursos adaptados al diseño universal de aprendizaje, una dinámica y organización del aula favorecedora de la inclusión, un proceso de evaluación formativo, la participación y la colaboración familiar en el aula y la promoción de un ambiente de aprendizaje equitativo e igualitario. Todo ello con el objetivo de desarrollar una cultura inclusiva que atienda a la diversidad de todo el alumnado (Takala, Pirttimaa y Törmänen, 2009).

Una educación inclusiva conlleva el uso de estrategias y metodologías didácticas y organizativas activas por parte de todo el profesorado (Azorín y Arnaiz, 2015), como el aprendizaje cooperativo (Johnson, Johnson y Holubec, 1999), la investigación-acción-participación (Ander-Egg, 2003), la tutoría entre iguales (Durán y Martínez, 2004), talleres y rincones, las redes de colaboración (Azorín y Muijs, 2018), las aulas invertidas (Altemueller y Lindquist, 
2017), la ludificación (Jiménez et al., 2019), el aprendizaje basado en problemas (Filippatou y Kaldi, 2010) o el aprendizaje-servicio (Gómez-Hurtado, Moya Maya y García-Rodríguez, 2019). Estas favorecen el desarrollo de prácticas en el aula que promueven la atención de todo el alumnado bajo los principios de igualdad, solidaridad, equidad y justicia social. El uso de estas estrategias y metodologías, junto con la creación de redes de colaboración, la utilización de recursos siguiendo los principios del diseño universal de aprendizaje (Azorín y Arnaiz, 2015) y el desarrollo de una organización escolar para la diversidad son elementos fundamentales para construir una escuela inclusiva, siendo la dirección escolar y el liderazgo inclusivo un factor importante en la promoción de dichos elementos (León, 2012) y la edificación de una cultura inclusiva (Valdés, 2018).

A partir de este marco teórico de referencia, el estudio que se presenta tiene como finalidad dar respuestas a preguntas tales como ¿qué papel tienen los maestros y las maestras de atención a la diversidad en el desarrollo de escuelas inclusivas?, ¿cuál es la formación de estos maestros y maestras?, ¿en qué estrategias se basan para desarrollar el apoyo?, ¿qué modelos de apoyo llevan a la práctica?, ¿qué recursos utilizan?

\section{Método}

Tomando en consideración las propiedades del discurso narrativo de los implicados en la práctica docente, se optó por el diseño de un estudio cualitativo (Flick, 2015) de corte transversal para investigar las dinámicas y las prácticas de los especialistas de atención a la diversidad. No obstante, también se han incorporado algunos datos de carácter cuantitativo que han matizado y facilitado la propensión panorámica de la información que perfila a los participantes. Desde un planteamiento descriptivo e interpretativo, este trabajo extiende las líneas de continuidad recogidas por García Prieto y DelgadoGarcía (2017).

Como objetivo general, nos planteamos describir las prácticas profesionales de los maestros de atención a la diversidad en Andalucía para construir una escuela inclusiva analizando el papel de los especialistas en atención a la diversidad, la dinámica y la organización del aula, estudiando las estrategias didácticas utilizadas para el apoyo y los modelos de este y conociendo los recursos utilizados por los docentes de atención a la diversidad.

\subsection{Participantes}

Este trabajo toma como población de referencia al profesorado especialista en pedagogía terapéutica ( 4644 personas) que imparte docencia en la comunidad autónoma de Andalucía durante el curso 2018-2019. La selección de sujetos se realiza a partir de un muestreo no probabilístico en el que se utilizan los siguientes criterios: la accesibilidad y la disposición a participar de manera voluntaria en el estudio, la categoría profesional (ser especialista en pedagogía 
terapéutica) y pertenecer a centros de educación infantil y primaria (públicos, privados o concertados) de Andalucía. Se accede a una muestra de 142 especialistas, la cual se corresponde con el mismo número de centros educativos participantes en el estudio (104 de titularidad pública, 36 concertados y 2 privados). La distribución de frecuencias entre las provincias andaluzas es la siguiente: 14 de Almería, 12 de Cádiz, 18 de Córdoba, 22 de Granada, 24 de Huelva, 11 de Jaén, 16 de Málaga y 25 de Sevilla. El 94,4\% de los participantes son mujeres y el 5,6\%, hombres, y su media de edad se sitúa en los 42,15 años. Respecto a la experiencia docente, es de 15,73 años de media. Por aula, indican que trabajan con una ratio de 2,70 alumnos con necesidades especiales de apoyo educativo, cifra próxima a la media de los centros públicos de la comunidad $(\bar{x}=3,61)$, pero alejada de la media de los centros privados $(\bar{x}=6,12)$ (Consejería de Educación y Deporte, 2019).

\subsection{Instrumentos de recogida de datos}

Como instrumento principal se utiliza una entrevista semiestructurada, diseñada ad hoc, compuesta por un total de 17 ítems de respuesta abierta y apoyada en los fundamentos de los protocolos de Kvale (2012), así como los de diseño e implementación de Merriam (2014), estructurada en siete dimensiones: perfil y formación; estrategias didácticas; recursos; dinámica y organización del aula; apoyo; satisfacciones y expectativas futuras, y obstáculos y dificultades. Para la validación del instrumento se recurre a la técnica de juicio de expertos (claridad y adecuación de contenido) mediante la revisión de dos docentes de la Universidad de Huelva especialistas en metodología de investigación. Por su parte, para valorar la confiabilidad del mismo instrumento, se recurre a una prueba piloto con diez docentes procedentes de la muestra seleccionada, a través de la cual se valora la dificultad de los ítems y el tiempo destinado a dar respuesta a estos. A partir de ambos casos, se realizaron ajustes menores relativos a la ortografía y la corrección de la formulación de tres cuestiones, puesto que el tiempo aproximado (35 minutos) se consideró adecuado.

En segundo lugar, se utiliza como técnica la observación directa y no participante, para que los acontecimientos se desarrollen de forma espontánea (Atkinson y Hammersley, 2008), y como instrumento se emplea un diario de investigación en el que se sintetizan los detalles y los registros anecdóticos que emergen de las situaciones en las que se desarrollan las entrevistas, así como otras conversaciones informales que se hayan podido mantener con los participantes. La estructura del diario se compone según diferentes dimensiones de análisis: comunicación no verbal, impresiones personales del investigador, anécdotas o incidentes críticos.

\subsection{Procedimiento y análisis de la información}

La administración de las entrevistas y el proceso de observación tuvo lugar entre el 16 de abril y el 14 de junio de 2019. Se informó a los participantes 
acerca de los objetivos y el sentido de la investigación, se solicitó su participación voluntaria y se garantizó el anonimato. Además, se devolvió toda la información a los participantes para verificar la fidelidad del discurso y asegurar los criterios éticos y la credibilidad del discurso.

Para el análisis de la información recopilada se utiliza el programa ATLAS. ti (versión 6), que garantiza una mayor exhaustividad en el procedimiento analítico. Las citas textuales utilizadas mantienen la siguiente codificación: «las estrategias o medidas llevadas a cabo afectan a los horarios, los materiales, las adaptaciones curriculares y las rutinas» 5:2 (16:17), donde "5» significa el número del protagonista (evidencia en entrevista u observación), «2» se refiere al número de cita dentro de ese documento y «(16:17)» indica el párrafo donde se inicia y se termina la cita.

\section{Resultados}

Una radiografía general de los datos de los perfiles muestra que el 78,9\% de los participantes objeto de estudio indican que tienen formación suficiente para atender a la diversidad. Dicha formación procede, en este orden, de cursos de formación del profesorado a través del Centro de Profesorado (CEP), entre los cuales resaltan la modalidad de formación en centros; el intercambio de experiencias docentes; las reuniones con los equipos de orientación educativa, los equipos directivos y los tutores; la asistencia a jornadas y seminarios de asociaciones y entidades privadas, o la realización de cursos en línea y consultas web.

Respecto al apoyo, el 69\% lo realiza en la combinación dentro-fuera del aula ordinaria. El $21,1 \%$ de docentes lo desarrolla siempre fuera, y solo el $9,9 \%$, exclusivamente en el aula ordinaria.

Sobre las concepciones del término diversidad, lo entienden como atender a las necesidades de manera individual. Para definirlo, las palabras que más se repiten son las siguientes: adecuar, adaptar y diferentes capacidades. Como evidencia, veamos dos definiciones generalizadas:

Conjunto de acciones para prevenir y dar respuestas a las necesidades educativas de todo el alumnado, tanto puntual como permanentemente. 3:1 (63:63)

Proporcionar los recursos personales y materiales que sean necesarios para alcanzar los objetivos de todos los alumnos y la adquisición de competencias. $31: 1(65: 65)$

En la práctica de aula, el 32,4\% del profesorado de apoyo trabaja contenidos distintos a los que imparten los tutores en el aula ordinaria. Una amplia mayoría (un 67,6\%) trabaja el contenido transversalmente mediante una labor de coordinación con los tutores.

Según los datos extraídos (no excluyentes), las necesidades concretas con mayor incidencia que se atienden habitualmente son las siguientes: 
- Trastorno del espectro autista (un 61,9\%).

- Trastorno del déficit de atención e hiperactividad (un 59,1\%).

- Dificultades de aprendizaje (dislexia, disgrafía, disortografía...) (un 56,3\%).

- Discapacidad intelectual (un 45,7\%).

Igualmente, consideran que la atención a la diversidad es una de las líneas de actuación prioritarias del centro, excepto un porcentaje situado en torno al $18 \%$ que lo justifica de la siguiente manera:

No es prioritario, hay que estar muy pendiente de que el profesorado se implique más en la atención a la diversidad de sus aulas, y no dejen que esa responsabilidad recaiga en los especialistas en pedagogía terapéutica y audición y lenguaje. $76: 3(80: 80)$

\subsection{Estrategias didácticas}

En la dinámica de aula se utilizan distintas estrategias y técnicas que atienden a la diversidad del alumnado, aunque depende de las singularidades, de los ritmos de aprendizaje o de si se llevan a cabo las mismas dentro o fuera del aula ordinaria. En líneas generales, se fomenta la participación del alumnado desde los agrupamientos flexibles, los grupos interactivos, las tertulias y los talleres.

— «Normalmente propongo agrupamientos flexibles, adaptaciones curriculares, apoyo individual, talleres y programas específicos» 6:7 (68:68).

— «Estrategias de aprendizaje cooperativo, a partir del asesoramiento a los tutores para su desarrollo en el aula ordinaria, y aplicación en gran grupo» 89:5 (71:71).

— «strategias de pensamiento (rutinas de pensamiento, autoinstrucciones...) para que el alumnado adquiera estrategias para enfrentarse a los problemas» 93:10 (84:84).

Algunos de los métodos y de las estrategias más empleados son: método Teacch, método Denver, trabajo cooperativo, trabajo colaborativo, tutorías entre iguales, técnicas de habilidades sociales y agrupamientos flexibles. También se describen los mismos en el marco de los programas específicos (dislexia, TDAH). Todos los que se utilizan tienen como finalidad atender al alumnado en función de sus necesidades.

En opinión de los participantes, estas estrategias afectan en mayor medida a los siguientes elementos:

1. A los horarios y su distribución.

2. A los agrupamientos del alumnado y a los materiales.

3. A los elementos y a la reformulación del currículo. 


\subsection{Recursos}

Según la opinión de los participantes, por recursos para atender a la diversidad se entiende que son todos aquellos medios y materiales plurales que abarcan todas las necesidades del aula. Se suelen encontrar desde aquellos más deliberativos hasta aquellos más convencionales; desde los que se utilizan sin necesidad de adaptarlos hasta los que se diseñan para una necesidad concreta; desde los que se presentan en un formato digital hasta otros impresos. En definitiva, deben ser funcionales y con un determinado propósito, aunque en ocasiones la ausencia de los mismos es una barrera para el trabajo diario en el aula, y también una posibilidad, puesto que abrimos la opción de crearlos y adaptarlos. Es decir, materiales y herramientas con un claro enfoque inclusivo.

Entre aquellos que más se utilizan suelen ser las agendas personalizadas, el material manipulativo, el material visual, los temarios adaptados y los pictogramas (digitales e impresos):

- «Materiales adaptados, fichas adaptadas a los niveles de los alumnos, diferentes juegos interactivos adaptados, ordenador táctil, mesas y sillas adaptadas para alumnado con discapacidad» 3:11 (93:93).

- «Cuento con recursos materiales, la mayoría son libros de texto y de actividades. Hay una falta grande de recursos» 108:10 (85:85).

Entre los recursos digitales encontramos las tecnologías de apoyo a la diversidad (TAD). Casi el 50\% de los especialistas utilizan aplicaciones y programas como Picto Selector, AraWord, ARASAAC o Yo soy visual:

— «La tecnología está presente, aunque se opta por trabajar con material en formato papel. En el aula contamos con un teclado adaptado, con una tablet y con un pulsador. El uso de estas tecnologías depende de las necesidades educativas que presente el alumnado» 49:14 (98:99).

\subsection{Dinámica y organización del aula}

La dinámica del aula variará en función de cada escolar y de la coordinación entre tutor y especialistas. Se puede llevar a cabo la atención de forma individualizada en los casos que lo requieran o en pequeños grupos que presenten la misma necesidad.

La secuencia más común con la que trabajan es la de asamblea, contenidos curriculares, juego libre y psicomotricidad.

— «La dinámica es diferente todos los días, no hay ningún día igual y depende de si están en el aula ordinaria o no» 122:14 (103:104).

— «utinas diarias (organización del tiempo y del espacio), trabajo académico (lengua, mates...), contenido del programa específico, juego» 8:16 (91:91). 
Por otro lado, en el aula de pedagogía terapéutica se atiende en pequeños grupos y cada sesión se estructura en tres momentos: «Primera parte: toma de contactos, saludos y conversaciones cercanas al alumnado. Segunda parte: desarrollo de los contenidos de los programas específicos de cada alumno o alumna. Tercera parte: reflexión sobre lo aprendido y juegos de cohesión grupal» 92:14 (106:108).

Con frecuencia se establece una actividad motivadora, después otra que requiere mayor atención y concentración, para finalizar de nuevo con una actividad lúdica que sirve para afianzar lo aprendido o como evaluación.

A propósito de la organización, el aula adopta distintas disposiciones. En forma de U, por rincones o por zonas diferenciadas. En cada una de ellas hay materiales adaptados a las diferentes necesidades del alumnado. El éxito del grupo depende del trabajo comunitario, y no del individual. De esta forma, la ayuda y colaboración entre compañeros es imprescindible.

— «Por grupos cooperativos, para incluir al alumnado con necesidades y en pequeños grupos de trabajo aprendemos juntos. Por grupos flexibles, donde en pequeños grupos y distinto nivel trabajamos el mismo contenido. De forma individualizada, para también atender al alumnado en su globalidad» 53:19 (110:111).

Conocer los intereses, las motivaciones y el estilo de aprendizaje es fundamental, pues esto lleva a distintos tipos de organización.

\subsection{El apoyo}

Se lleva a cabo desde diversos planteamientos. En educación infantil, preferentemente dentro del aula - es más común — y con la misma actividad que el resto de la clase (Nadal Morant, Grau Rubio y Peirats Chacón, 2016). En educación primaria, fuera del aula ordinaria en grupos reducidos, reforzando áreas comunes o realizando otras actividades que posibilitan trabajar contenidos curriculares.

Cuando se realiza fuera, se apoya y se refuerza al alumnado antes y después de los contenidos que se desarrollan en el aula ordinaria.

— «En mi centro el apoyo se realiza fuera del aula en primaria y dentro del aula en infantil» 25:18 (91:91).

— «El apoyo se lleva a cabo dentro del aula ordinaria, trabajando los mismos contenidos que el tutor, pero desde un punto de trabajo más bajo para reforzar el aprendizaje de aquellos alumnos que lo necesiten» 121:20 (113:113).

— «En la mayoría de los casos, trabajamos fuera del aula ordinaria contenidos concretos de los programas específicos que desarrollamos con el alumnado» 53:19 (110:111). 
Para realizar el apoyo dentro del aula se deben tener en cuenta diferentes factores, como el número de alumnado al que se atiende, la coordinación del pedagogo terapéutico (PT) y el tutor, la adaptación de programaciones por parte del tutor, la organización del aula ordinaria y el desfase curricular. Es necesario llevar a cabo las adaptaciones pertinentes, tanto en metodología como en actividades, contenidos y, en su caso, objetivos para que el currículo y la dinámica del alumnado sean lo más parecidos posible a los del resto de su grupo clase.

Veamos algunas evidencias:

— «No tiene sentido realizar el apoyo dentro del aula en el caso en el que el maestro o la maestra PT está trabajando con el escolar algo totalmente diferente a lo que se aprende en la clase ordinaria, y sobre todo en un rincón apartado» 103:22 (118:118).

— «Soy partidaria del apoyo dentro del aula ordinaria y trabajar los mismos contenidos que el resto. Lo idóneo sería que tanto el docente del área como el maestro o la maestra de pedagogía terapéutica trabajasen coordinados dentro del aula ordinaria, para que no hubiera ningún tipo de discriminación o desigualdad» 19:26 (125:125).

El enfoque inclusivo, por tanto, no es un proceso que se lleve a cabo de un día para otro, sino que requiere de cambios de procedimientos, recursos y actitudes a la hora de trabajar en el aula ordinaria. Seguimos viendo más discursos y deseos que prácticas, por lo cual coincidimos con las conclusiones de otros estudios actuales que analizan percepciones y prácticas (Vallejo et al., 2019; Colmenero, Pegalajar y Pantoja, 2019).

De los resultados obtenidos podemos destacar que se mantiene la combinación de los cuatro modelos establecidos por Parrilla (1996) en la práctica educativa hacia la inclusión. De acuerdo con Torres y Fernández-Batanero (2015), la maestra o el maestro de pedagogía terapéutica tiene funciones de apoyo, principalmente fuera del aula con el alumnado con necesidades educativas especiales y, en segundo lugar, de coordinación con el docente tutor. Sandoval, Simón y Echeita (2012), entre otros, defienden el modelo de apoyo curricular para una educación de calidad con equidad.

— «El apoyo se lleva a cabo de forma adecuada, habiendo una buena coordinación entre el tutor y el profesor de apoyo y programando asumiendo responsabilidades de forma conjunta para dar una buena respuesta al alumnado que lo precisa» 89:36 (134:134).

— «El especialista debe trabajar los mismos contenidos que el tutor en el aula ordinaria, para ello se organiza el aula y se revisan los niveles de competencia, para terminar llevando a cabo un currículum de forma colaborativa» $18: 25(102: 102)$. 
Rappoport y Echeita (2018) señalan algunas de las limitaciones que tiene un modelo individual y terapéutico centrado en el profesional de apoyo, por ejemplo: efecto «delegación» de responsabilidad y una atención más limitada.

\subsection{Satisfacciones y expectativas futuras}

La satisfacción va unida con la consecución de los logros propuestos, pero en otras ocasiones tiene que ver con cuestiones epistemológicas.

— «No todo logro puede ser medido de la misma forma. Hay alumnado con más posibilidades de mejora que otro, con lo que los logros y objetivos a alcanzar están personalizados. El grado de satisfacción con el alumnado es excelente, con los medios (materiales y humanos) que nos aporta el sistema podría calificarlo de mejorable» 101:29 (104:104).

- «En este ámbito hay que plantearse expectativas a corto plazo, cuando el alumnado lo alcanza se siente una satisfacción plena» 96:39 (164:164).

Entre estos especialistas la satisfacción es positiva, aunque a veces perciben que todo el trabajo llevado a cabo no queda bien reflejado.

Siempre intentan que se alcancen los máximos logros posibles, pero no depende únicamente del especialista, sino también del tutor, del propio alumnado, de la familia y del centro, entre otros. Los resultados alcanzados suelen verse a largo plazo y consideran que hay un largo camino por recorrer, sobre todo desde la Administración.

— «Los maestros en las aulas trabajan duro, y con más recursos personales la atención a la diversidad terminaría siendo más real y no tan burocrática» 74:38 (132:132).

Las expectativas apuntan hacia un interés en fomentar el desarrollo integral del alumnado, la aceptación, la autoestima y el autoconcepto. En general, se trata de prepararse para su realidad en la sociedad.

\subsection{Obstáculos y dificultades}

Entre los problemas más destacables que se encuentra el pedagogo terapéutico (también los tutores) son, por este orden:

1. Escasez de tiempo para atender a las necesidades individuales.

2. Número elevado de alumnado (censado en Séneca).

3. Carencia de apoyo y de implicación y colaboración familiar.

4. Coordinación deficiente del tutor con el especialista.

5. Dificultad en la organización de horarios de infantil y primaria.

6. Poca sensibilidad por parte de algunos tutores o tutoras.

7. Falta de recursos materiales. 
Es recurrente destacar evidencias como:

— «La principal dificultad es el número de escolares con dificultades. Las sesiones de clases son de cuarenta y cinco minutos. Con tantas especialidades (inglés, francés, educación física, música, religión, asignaturas bilingües) los tutores están poco tiempo con los alumnos. Se trata de horarios donde el tutor no permanece dos sesiones seguidas con su alumnado» 23:42 (176:178).

En determinados centros educativos el bilingüismo puede llegar a ser una rémora en la mejora de la atención a la diversidad del alumnado. También se pone de manifiesto la postura o la rigidez de la Administración hacia la atención a la diversidad (Echeita, 2017), las exigencias del currículo y la presión de los resultados en las evaluaciones externas, con el fin de crear comparativas y estadísticas.

\section{Discusión y conclusiones}

La formación de los profesionales en atención a la diversidad y de los tutores es uno de los aspectos que se remarcan como fundamentales para enseñar desde un enfoque inclusivo (Collado Sanchis et al., 2020). Los profesionales de atención a la diversidad andaluces consideran, en su mayoría, que tienen la formación necesaria para atender a la diversidad del alumnado, sin embargo remarcan que el profesorado tutor debería cuidar más su formación permanente.

La construcción de una cultura inclusiva sigue siendo uno de los objetivos prioritarios de las escuelas de todo el mundo (Echeita et al., 2019). Las funciones de apoyo desarrolladas en las escuelas se deben realizar desde un enfoque inclusivo, en el cual el maestro de atención a la diversidad pertenece más a la red de colaboración creada (Biedma y Moya, 2015; Salas, 2016). Este enfoque inclusivo no es una realidad en la mayoría de los casos de nuestro estudio, dado que los apoyos se realizan tanto fuera como dentro del aula, e incluso de manera mixta. Así pues, se hace visible la multiplicidad de modelos relativos a la práctica profesional docente para atender a la inclusión educativa, entre los que se potencian especialmente el apoyo colaborativo/ individual y el modelo de apoyo curricular (Parrilla, 1996). Coincidiendo con otros estudios, llegamos a la conclusión de que la inclusión escolar es aún más un deseo que una práctica (Vallejo et al., 2019; Colmenero et al., 2019).

De acuerdo con Soldevila et al. (2017), para que este modelo de apoyo inclusivo sea una realidad, se necesitarán algunas realidades y cambios: promocionar un rol encaminado a apoyar a la inclusión, potenciar la corresponsabilización del niño con discapacidad para su vida en la escuela, facilitar la coordinación entre la tutora y las maestras profesionales de apoyo y crear estructuras y figuras que estimulen la inclusión. 
La importancia de desarrollar metodologías activas para atender a la diversidad del alumnado de un modo inclusivo es otra conclusión que se desprende del análisis presentado (Azorín y Arnaiz, 2015). No hay una estrategia única para el desarrollo de prácticas inclusivas, sino propuestas alternativas en función de las necesidades de cada caso (García Prieto y Delgado-García, 2017). El diseño universal de aprendizaje aboga por el principio de inclusión para atender a la diversidad del alumnado y remarca la importancia de los recursos (funcionales y para todos) en el desarrollo de este proceso de cambio hacia la inclusión escolar.

Sin duda alguna, la organización del aula es otro aspecto que tratar a la hora de construir un entorno inclusivo. La flexibilidad en la organización es el aspecto clave para poder adaptarse a las demandas existentes en el aula.

El profesorado especialista en atención a la diversidad liga estrechamente su satisfacción con los logros obtenidos haciendo referencia de nuevo a la importancia del trabajo colaborativo entre todos los miembros de la comunidad educativa (Ainscow, 2020). Asimismo, también se reconocen obstáculos.

Desde la perspectiva de los especialistas en atención a la diversidad en Andalucía, la inclusión es un reto en el cual tienen un papel fundamental como apoyo a toda la comunidad educativa.

Entre las limitaciones, cabe destacar la necesidad de tomar con prudencia las generalizaciones de la información analizada, dado que la muestra no alcanza una representatividad amplia de la población. A propósito de la prospectiva, sería oportuno profundizar en algunas de las variables que emergen del contenido de las dimensiones de estudio, como la formación específica que posee el profesorado.

\section{Referencias bibliográficas}

Ainscow, M. (2020). Promoting inclusion and equity in education: Lessons from international experiences. Nordic Journal of Studies in Educational Policy, 6(1), $7-16$. <https://doi.org/10.1080/20020317.2020.1729587>

Altemueller, L. y Lindquist, C. (2017). Flipped classroom instruction for inclusive learning. British Journal of Special Education, 44(3), 341-358. $<$ https://doi.org/10.1111/1467-8578.12177>

Ander-EgG, E. (2003). Repensando la investigación-acción-participativa. Buenos Aires: Lumen.

Arnaiz, P. y Caballero, C. (2020). Estudio de las Aulas Abiertas Especializadas como Medida Específica de Atención a la Diversidad. Revista Internacional de Educación para la Justicia Social, 9(1), 191-210. <https://doi.org/10.15366/riejs2020.9.1.009>

Atrinson, P. y Hammersley, M. (2008). Etnografia: Métodos de investigación. Barcelona: Paidós Ibérica.

Azorín, C.M. y Arnaiz, P. (2015). Una experiencia de innovación en educación primaria: Medidas de atención a la diversidad y diseño universal del aprendizaje. Tendencias Pedagógicas, 22, 9-30. Recuperado de <https://revistas.uam.es/tenden ciaspedagogicas/article/view/2039>. 
Azorín, C. y Muijs, D. (2018). Redes de colaboración en educación: Evidencias recogidas en escuelas de Southampton. Profesorado: Revista de Curriculum y Formación del Profesorado, 22(2), 7-27. <https://doi.org/10.30827/profesorado.v22i2.7845>

Biedma, P.E. y Moya, A. (2015). La intervención del Profesorado de Apoyo a la Integración dentro del aula ordinaria en Huelva capital: Hacia una escuela inclusiva. Revista Nacional e Internacional de Educación Inclusiva, 8(2), 153-170. Recuperado de <https://revistaeducacioninclusiva.es/index.php/REI/article/ view/112/109>.

Brownell, M.T.; Ross, D.D.; Colón, E.P. y McCallum, C.L. (2005). Critical features of special education teacher preparation: A comparison with general teacher education. The Journal of Special Education, 38(4), 242-252. <https://doi.org/10.1177/00224669050380040601>

Collado Sanchis, A.; Tárraga Mínguez, R.; Lacruz Pérez, I. y Sanz Cervera, P. (2020). Análisis de actitudes y autoeficacia percibida del profesorado ante la educación inclusiva. Educar, 56(2), 509-523. <https://doi.org/10.5565/rev/educar.1117>

Colmenero, M.J.; Pegalajar, M.C. y Pantoja, A. (2019). Percepción del profesorado sobre prácticas docentes inclusivas en alumnado con discapacidades graves y permanentes. Cultura y Educación, 31(3), 542-575. <https://doi.org/10.1080/11356405.2019.1630952>

CONSEJERÍA DE EDUCACIÓN Y DePORTE (2019). Estadística sobre recursos y utilización del sistema educativo andaluz, a excepción del universitario. Junta de Andalucía. Recuperado de <https:/www.juntadeandalucia.es/servicios/estadistica-cartografia/ actividad/detalle/175114/175500.html>.

Corujo, C.; Gallego, C. y Jiménez, A. (2018). Otra forma de desarrollar el apoyo inclusivo: Los Grupos de Apoyo Mutuo. Revista de Investigación en Educación, 16(2), 106-120. Recuperado de <http://reined.webs.uvigo.es/index.php/reined/ article/view/341>.

Durán, D. y Martínez, I. (2004). Tutoría entre iguales. Innovación Educativa, 75 , 63-68.

Echeita, G. (2017). Educación inclusiva: Sonrisas y lágrimas. Aula Abierta, 46, 17-24. <https://doi.org/10.17811/rifie.46.2.2017.17-24>

Echeita, G.; Márquez, C.; Sandoval, M. y Simón, C. (2019). El desempeño profesional del profesorado de apoyo y sus aportaciones al desarrollo de una educación inclusiva. Publicaciones, 49(3), 251-266.

<https://doi.org/10.30827/publicaciones.v49i3.11412>

Filippatou, D. y Kaldi, S. (2010). The Effectiveness of Project-Based Learning on Pupils with Learning Difficulties Regarding Academic Performance, Group Work and Motivation. International Journal of Special Education, 25(1), 17-26. <https://doi.org/10.1177/2158244020938702>

Flick, U. (2015). El diseño de investigación cualitativa. Madrid: Morata.

GAIRÍN-SALlán, J. (1998). Estrategias organizativas en la atención a la diversidad. Educar, 22, 239-267.

<https://doi.org/10.5565/rev/educar.360>

García Prieto, F.J. y Delgado-García, M. (2017). Estrategias de enseñanza como respuesta a la diversidad: Concepciones y prácticas del pedagogo terapéutico. Revista de Educación Inclusiva, 10(1), 103-116. Recuperado de <http://hdl.handle. net/11441/68839>. 
Gómez-Hurtado, I.; Moya Maya, A. y García-Rodríguez, M.P. (2019). Aprendizaje servicio en la formación inicial de docentes en la Universidad de Huelva: El proyecto INCLUREC. REICE: Revista Iberoamericana sobre Calidad, Eficacia y Cambio en Educación, 18(1), 105-123.

$<$ https://doi.org/10.15366/reice2020.18.1.006>

Instrucciones de 8 de marzo de 2017, de la Dirección General de Participación y Equidad, por las que se actualiza el protocolo de detección, identificación del alumnado con necesidades específicas de apoyo educativo y organización de la respuesta educativa. Sevilla. Junta de Andalucía. Consejería de Educación. Dirección General de Participación y Equidad.

Jiménez, C.R.; Navas-Parejo, M.R.; Villalba, M.J.S. y Campoy, J.M.F. (2019). El uso de la gamificación para el fomento de la educación inclusiva. International Journal of New Education, 2(1), 39-59. <https://doi.org/10.24310/IJNE2.1.2019.6557>

Johnson, D.W.; Johnson, R.T. y Holubec, E.J. (1999). El aprendizaje cooperativo en el aula. Recuperado de <https:/www.guao.org/sites/default/files/biblioteca/ El\%20aprendizaje\%20cooperativo\%20en\%20el\%20aula.pdf>.

Kvale, S. (2012). Las entrevistas en investigación cualitativa. Madrid: Morata.

León, M.J. (2012). El Liderazgo para y en la Escuela Inclusiva. Educatio Siglo XXI, 30(1), 133-160. Recuperado de <https://revistas.um.es/educatio/article/view/149181>.

Liasidou, A. y Antoniou, A. (2013). A special teacher for a special child?: (Re) considering the role of the special education teacher within the context of an inclusive education reform agenda. European Journal of Special Needs Education, 28(4), 494-506. <https://doi.org/10.1080/08856257.2013.820484>

Merriam, S.B. (2014). Case Studies as Qualitative Research: S. B. Merriam. Qualitative Research: A Guide to Design and Implementation (pp. 26-43). San Francisco: Jossey-Bass.

Muntaner, J.; Roselló, M.R. e Iglesia, B. de la (2016). Buenas prácticas en Educación Inclusiva. Educatio Siglo XXI, 34(1), 31-50. <https://doi.org/10.6018/j/252521>

Nadal Morant, M.J.; Grau Rubio, C. y Peirats Chacón, J. (2016). Análisis y valoración del modelo inclusivo en los sistemas de apoyo de centros de infantil y primaria. Educatio Siglo XXI, 34(3), 161-180. <http://dx.doi.org/10.6018/j/276001>

Noguera, J.A. y Hernández, M.A. (2019). Inclusión educativa: una mirada crítica al perfil del maestro especialista en pedagogía terapéutica. En R. Moreno y A. TEJADA. IV Congreso internacional virtual sobre La Educación en el Siglo XXI (pp. 43-56). España, Almería. Recuperado de <https://sid.usal.es/idocs/F8/ FDO27377/iAccessibility_15.pdf>.

Parrilla, M.A. (1996). Apoyo interno: Modelos y funciones. En M.A. Parrilla (ed.). Apoyo a la escuela: Un proceso de colaboración (pp. 81-109). Bilbao: Mensajero.

Rappoport, S. y Echeita, G. (2018). El docente, los profesionales de apoyo y las prácticas de enseñanza: Aspectos clave en la configuración de aulas inclusivas. Perspectiva Educacional: Formación de Profesores, 57(3), 3-27. <http://dx.doi.org/10.4151/07189729-vol.57-iss.3-art.740>

Salas, M. (2016). Reflexiones en torno a los maestros de pedagogía terapéutica. Padres y Maestros: Journal of Parents and Teachers, 365, 6-11. <https://doi.org/10.14422/pym.i365.y2016.001> 
Sandoval, M.; Simón, C. y Echeita, G. (2012). Análisis y valoración crítica de las funciones del profesorado de apoyo desde la educación inclusiva. Revista de Educación, número extraordinario, 117-137.

$<$ http://doi.org/10.4438/1988-592X-RE-2012-EXT-209>

Soldevila, J.; Pérez, I.; Naranjo, M. y Muntaner, J.J. (2017). El profesor de apoyo del modelo de integración a la inclusión educativa. En A. RodríGuez-MarTín (comp.). Prácticas innovadoras inclusivas: Retos y oportunidades (pp. 853860). Oviedo: Universidad de Oviedo.

Takala, M.; Pirttimaa, R. y Törmänen, M. (2009). Research section: Inclusive special education: the role of special education teachers in Finland. British Journal of Special Education, 36(3), 162-173. <https://doi.org/10.1111/j.1467-8578.2009.00432.x>

Torres, J.A. y Fernández-Batanero, J.M. (2015). Promoviendo escuelas inclusivas: Análisis de las percepciones y necesidades del profesorado desde una perspectiva organizativa, curricular y de desarrollo profesional. Revista Electrónica Interuniversitaria de Formación del Profesorado, 18(1), 177-200. $<$ https://doi.org/10.6018/reifop.18.1.214391>

VALDÉs, R. (2018). Liderazgo inclusivo: La importancia de los equipos directivos en el desarrollo de una cultura de la inclusión. IE: Revista de Investigación Educativa de la Rediech, 9(16), 51-66. $<$ https://doi.org/10.33010/ie_rie_rediech.v9i16.73>

Vallejo, M.; Torres-Soto, A.; Curiel-Marín, E. y Campillo-Drieguez, O. (2019). Percepción y prácticas docentes para una educación inclusiva. Psicologia em Pesquisa, 13(3), $26-47$.

<https://doi.org/10.34019/1982-1247.2019.v13.27718> 